\title{
Improved flavor symmetry in Kogut-Susskind fermion actions
}

\author{
K. Orginos, ${ }^{\mathrm{a} *}$ R. Sugar, ${ }^{\mathrm{b}}$ and D. Toussaint ${ }^{\mathrm{a}}$

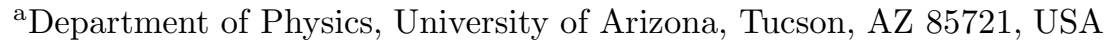 \\ ${ }^{\text {b} D e p a r t m e n t ~ o f ~ P h y s i c s, ~ U n i v e r s i t y ~ o f ~ C a l i f o r n i a, ~ S a n t a ~ B a r b a r a, ~ C A ~ 93106, ~ U S A ~}$
}

We study improved Kogut-Susskind fermion actions focusing on flavor symmetry restoration. Several variants of fat actions suitable for dynamical simulations are considered, including an action with no tree level $O\left(a^{2}\right)$ errors. The spectrum of all the pions is computed and used as a measure of flavor symmetry violation. Finally, the Naik term is introduced to restore rotational symmetry.

\section{INTRODUCTION}

Dynamical fermion simulations are still one of the challenging problems in lattice QCD. Recently, a lot of work has been devoted to developing improved lattice fermion actions. In this paper, we report our findings on improved KogutSusskind fermions. In particular, we focus on the issue of flavor symmetry, and the construction of an action suitable for dynamical simulations.

Kogut-Susskind (KS) fermions have $O\left(a^{2}\right)$ lattice artifacts, in contrast to the Wilson fermions which have $O(a)$. They represent four degenerate quark flavors with a $U(4) \times U(4)$ symmetry in the continuum. This symmetry is broken down to a $U(1) \times U(1)$ on the lattice. This remnant symmetry protects against additive mass renormalizations. The $U(4) \times U(4)$ symmetry breaking manifests itself in mass splittings in the pion spectrum. There is only one Goldstone pion associated with the exact $U(1)$ chiral symmetry, while the rest of the fifteen pions [1] are non-Goldstone massive particles.

The first step towards improving the KS action was made by Naik. He introduced the so called "Naik term" $\left(-\frac{1}{6} \Delta_{\mu}^{3}\right)$, which eliminates a tree level $O\left(a^{2}\right)$ artifact, improving the Lorenz symmetry (dispersion relation). The remaining order $O\left(a^{2}\right)$ artifacts are the $U(4) \times U(4)$ breaking terms. As Lepage [2] pointed out, these terms

\footnotetext{
*Presented by K. Orginos. Supported by the US DOE and NSF. Computations were done at ORNL, PSC, NCSA, $\mathrm{BU}$, and NERSC as part of the MILC collaboration.
}

are due to couplings to high momentum gluons, which cause scattering of lattice quarks among the corners of the Brillouin zone. These scatterings correspond to spin-flavor transformations of the physical quark field, causing the breaking of the symmetry. Thus, the way to reduce the symmetry breaking is to smear [3, 5, 8 ] the quarkgluon vertex, which suppresses the couplings to high momentum gluons. Recently, it was shown by Lepage [8] that the one gluon exchange interaction at high momentum is identical to 4fermi $U(4) \times U(4)$ breaking terms. Consequently, these effects can be canceled by either appropriate smearing of the quark-gluon vertex, so that high momentum gluons decouple, or by including in the action appropriate 4 -fermi terms.

\section{ACTION CONSTRUCTION}

Since the $U(4) \times U(4)$ symmetry breaking occurs due to coupling to high momentum gluons, we need to modify the quark-gluon vertex in order to decouple them. The coupling of quarks to a gluon $A_{\mu}$ with any of its transverse momentum components $p_{\nu}=\pi / a$ causes quark scattering among the corners of the Brillouin zone. Couplings to gluons with longitudinal momentum $p_{\mu}=\pi / a$ vanish due to cancellations between the forward and the backward part of the Dirac operator, consequently we do not need to worry about them. There are 3 independent couplings to high transverse momentum gluons $\left(V_{1}, V_{2}, V_{3}\right)$. We can eliminate them by modifying the gauge link used 
in the lattice derivative as following:

$$
\begin{aligned}
U_{\mu}(x) & \rightarrow c_{1} U_{\mu}(x)+\sum_{\nu}\left[w_{3} S_{\mu \nu}^{(3)}(x)+\right. \\
& \left.+\sum_{\rho}\left(w_{5} S_{\mu \nu \rho}^{(5)}(x)+\sum_{\sigma} w_{7} S_{\mu \nu \rho \sigma}^{(7)}(x)\right)\right]
\end{aligned}
$$

$$
\begin{aligned}
& S_{\mu \nu}^{(3)}(x)=U_{\nu}(x) U_{\mu}(x+\hat{\nu}) U_{\nu}^{\dagger}(x+\hat{\mu}) \\
& S_{\mu \nu \rho}^{(5)}(x)=U_{\nu}(x) S_{\mu \rho}^{(3)}(x+\hat{\nu}) U_{\nu}^{\dagger}(x+\hat{\mu}) \\
& S_{\mu \nu \rho \sigma}^{(7)}(x)=U_{\nu}(x) S_{\mu \rho \sigma}^{(5)}(x+\hat{\nu}) U_{\nu}^{\dagger}(x+\hat{\mu})
\end{aligned}
$$

It is not difficult to see that the above set of terms is the simplest needed to eliminate completely couplings to high momentum gluons.

In the weak coupling limit, the couplings $\left(V_{1}, V_{2}, V_{3}\right)$ to the gauge field with one, two or three of the transverse momentum components $\pm \pi / a$ can be expressed as functions of the staple couplings $w_{3}, w_{5}, w_{7}$, and the single link coupling $c_{1}$ :

$$
\begin{aligned}
& V_{1}=c_{1}+2 w_{3}-8 w_{5}-48 w_{7} \\
& V_{2}=c_{1}-2 w_{3}-8 w_{5}+48 w_{7} \\
& V_{3}=c_{1}-6 w_{3}+24 w_{5}-48 w_{7}
\end{aligned}
$$

The overall normalization condition

$c_{1}+6 w_{3}+24 w_{5}+48 w_{7}=1$

is used to ensure that the total coupling to the nearest neighbor in the free field limit is one. The solution to the equations $V_{1}=0, V_{2}=0, V_{3}=0$ is $c_{1}=2 w_{3}=8 w_{5}=48 w_{7}=1 / 8$. This set of parameters defines our "Fat7" action. The "Fat5" action is constructed by using paths up to length 5, minimizing the maximum of the couplings $|V|$. The "Fat5" couplings are $c_{1}=2 w_{3}=8 w_{5}=1 / 7$, $w_{7}=0$, which give $\left|V_{1}\right|=\left|V_{2}\right|=\left|V_{3}\right|=1 / 7$. Adding the Naik term to the "Fat7" action results in an action with no tree level $O\left(a^{2}\right)$ errors and improved flavor symmetry. Unfortunately, the extra couplings introduced to fix the high momentum behavior introduce a low momentum artifact $\sim A_{\mu} k_{\nu}^{2} a^{2}$. In order to correct this new artifact, one has to introduce a new term discovered by Lepage [8]. This new term is a double staple

$S_{\mu \nu}^{(L)}(x)=U_{\nu}(x) S_{\mu \nu}^{(3)}(x+\hat{\nu}) U_{\nu}^{\dagger}(x+\hat{\mu})$ with weight $w_{L}$. Cancellation of the new artifact occurs, if $w_{3} \rightarrow w_{3}-6 w_{L}$ and $w_{L}=-1 / 16$. Note that because the new term couples to a distance 2 in the $\nu$ direction, at momentum $p_{\nu}=\pi / a$ it acts as a single link term. For this reason, the above change of couplings preserves the normalization condition and keeps the couplings $V_{1}, V_{2}$, and $V_{3}$ zero. Adding the Naik term, $\left(-\frac{1}{6} \Delta_{\mu}^{3}\right)$ we get an action which we call "Asq", that has errors of $O\left(a^{4}, g^{2} a^{2}\right)$. All the above actions can be tadpole improved. For the tadpole improved actions, we append the suffix "tad" to the names above.

Motivated by the success of RG inspired APE smeared actions in the work of DeGrand, Hasenfratz and collaborators [5], we also decided to approximate APE smeared actions as a set of paths with appropriate weights. This is necessary, if we want to use such actions in dynamical simulations. In [7] we show how this approximation can be done expanding in powers of the APE smearing parameter $\alpha$. For one APE smearing with $\alpha=.25$, and to leading order in $\alpha$ we get the action we call "OFUN25" (once fattened unitarized $\alpha=.25)$. The fat link for this action is:

$U_{\mu} \rightarrow U_{\mu}+\frac{\alpha}{2} \sum_{\nu}\left(S_{\mu \nu}^{(3)}-U_{\mu} S_{\mu \nu}^{(3) \dagger} U_{\mu}\right)$.

\section{SIMULATIONS AND RESULTS}

Our action tests are focused on the flavor symmetry restoration, since we have established in our previous studies [6] that the Naik term improves Lorenz symmetry adequately for the lattice spacings used in current simulations. Furthermore, the Naik term has little effect on flavor symmetry. Thus, in most of the smearing variants we use, we do not include the Naik term. We include the Naik term into "Asq" and "Asqtad", since these actions are the candidates for an action with both improved flavor and rotational symmetry. The flavor symmetry breaking is estimated by the splittings in the pion spectrum. Spectroscopy is done on the same set of lattices (sample sizes range from 48 to 60 ) for all the actions tested. We use $12^{3} \times 32 \beta=7.3$ lattices with two dynamical quark masses $m=.020$ and $m=.040$, and $16^{3} \times 48 \beta=7.5$ lattices with dynamical quark masses $m=.015$ and $m=.030$. 


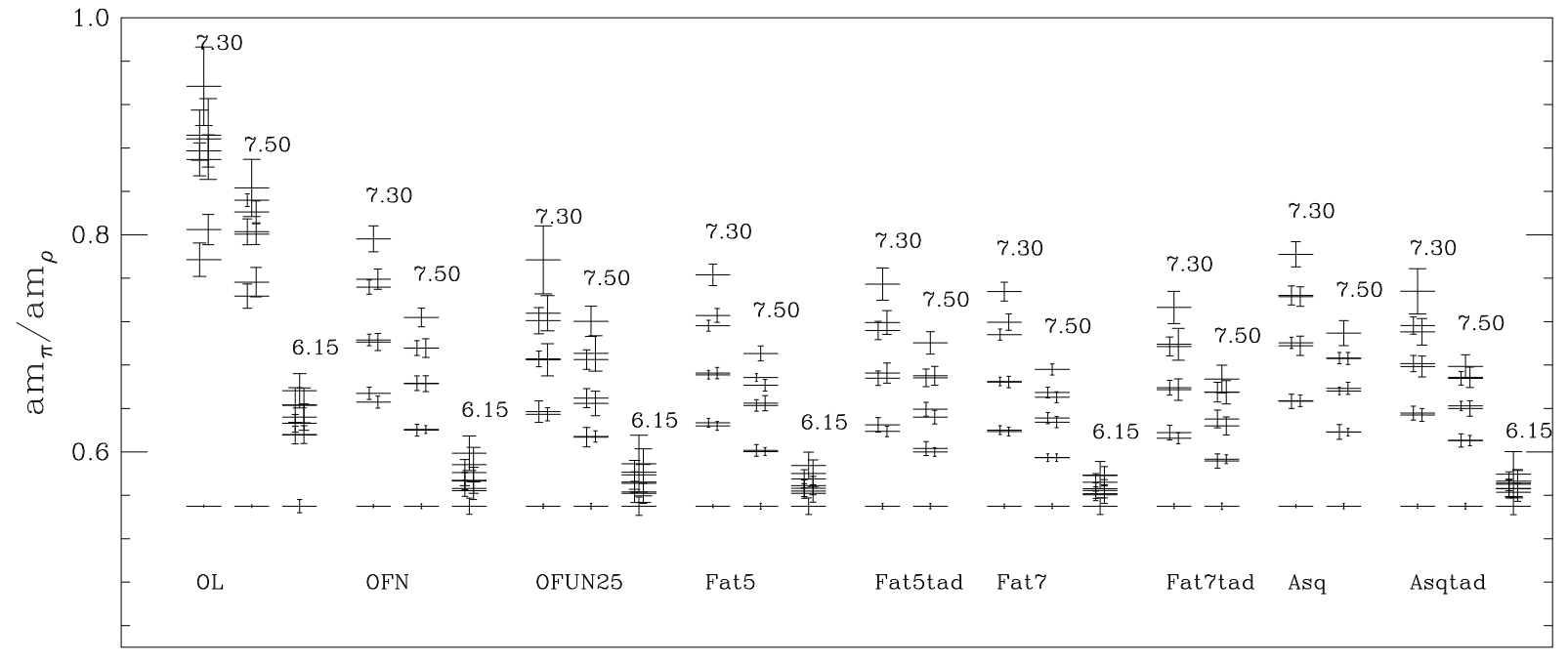

Figure 1. Interpolated pion masses for all the actions tested. The highest level is the flavor singlet pion while the lower is the Goldstone pion. The first doublet is the local non-Goldstone pion (flavor structure $\left.\gamma_{0} \gamma_{5}\right)$ and the $\gamma_{i} \gamma_{5}$ pion (right). The second is the $\gamma_{0} \gamma_{i}($ left $)$ and $\gamma_{i} \gamma_{j}$ pion. The third is the $\gamma_{0}($ left $)$ and $\gamma_{i}$ pion.

The gauge action is 1-loop Symanzik improved and the sea quark action is Kogut-Susskind with a staple and a Naik term [6,7]. We also use a set of $32^{3} \times 64$ quenched lattices at $\beta=6.15$ with Wilson gauge action. The pion spectra are computed for the two different masses in the case of the dynamical lattices and for $m=.010$ and $m=0.020$ in the case of the quenched lattices. We then interpolate the results to $m_{\pi} / m_{\rho}=.55$. The interpolated spectra are shown in Figure 1. In this figure, we also show for comparison the spectrum of the standard KS action (OL) and the spectrum of the action used for the dynamical quarks(OFN). Our results in Figure 1 show that there is a significant improvement of the flavor symmetry as we increase the suppression of the high momentum gluon couplings. The highest improvement is observed for the "Fat7tad" and "Asqtad" actions where couplings are set to zero at tree level. For all the actions the tadpole variant is better than the non-tadpole. The approximately reunitarized action "OFUN25" is slightly better than the "OFN". In all cases the spectrum exhibits the degeneracies suggested in 9]. For a detailed error analysis that better justifies our claims see our recent publication [7].
Although the differences in flavor symmetry improvement among the various highly smeared actions seem small, we favor "Asqtad" on theoretical grounds $\left(O\left(a^{4}, g^{2} a^{2}\right)\right.$ errors), and on our estimation that it only costs a factor of 2-3 for dynamical simulations with light quarks. We believe that such an action will be useful in realistic projects with dynamical quarks.

\section{REFERENCES}

1. M.F.L. Golterman, Nucl. Phys. B 273 (1986) 663.

2. G.P. Lepage, Nucl. Phys. (Proc. Suppl.) 60A (1998) 267.

3. T. Blum et al., Phys. Rev. D 55 (1997) 1133.

4. J.F. Lagae, D.K. Sinclair, hep-lat/9806014; Nucl. Phys. (Proc. Suppl.) 63 (1998) 892.

5. T. DeGrand, A. Hasenfratz and T. Kovacs, Phys. Lett. 420B (1998) 97.

6. K. Orginos, D. Toussaint Phys. Rev. D 59 (1999) 014501; Nucl. Phys. (Proc. Suppl.) 73 (1999) 909.

7. K. Orginos, R. Sugar, D. Toussaint Phys. Rev. D 60 (1999) 054503.

8. G.P. Lepage, Phys. Rev. D 59 (1999) 074502.

9. W. Lee, S. Sharpe hep-lat/9905023. 